\title{
Positive solutions for nonlinear parametric singular Dirichlet problems
}

\author{
Nikolaos S. Papageorgiou \\ Department of Mathematics, National Technical University \\ Zografou Campus, Athens 15780, Greece \\ Institute of Mathematics, Physics and Mechanics \\ Jadranska 19, 1000 Ljubljana, Slovenia \\ npapg@math.ntua.gr \\ Vicenţiu D. Rădulescu* \\ Institute of Mathematics, Physics and Mechanics \\ Jadranska 19, 1000 Ljubljana, Slovenia \\ Faculty of Applied Mathematics \\ AGH University of Science and Technology, al. \\ Mickiewicza 30, 30-059 Kraków, Poland \\ Institute of Mathematics \\ "Simion Stoilow" of the Romanian Academy \\ P.O. Box 1-764, 014700 Bucharest, Romania \\ vicentiu.radulescu@imar.ro \\ Dušan D. Repovš \\ Faculty of Education and Faculty of Mathematics and Physics \\ University of Ljubljana, 1000 Ljubljana Slovenia \\ Institute of Mathematics, Physics and Mechanics \\ Jadranska 19, 1000 Ljubljana, Slovenia \\ dusan.repovs@guest.arnes.si \\ Received 18 February 2018 \\ Accepted 13 July 2018 \\ Published 10 May 2019 \\ Communicated by Ari Laptev
}

We consider a nonlinear parametric Dirichlet problem driven by the $p$-Laplace differential operator and a reaction which has the competing effects of a parametric singular term and of a Carathéodory perturbation which is $(p-1)$-linear near $+\infty$. The problem is uniformly nonresonant with respect to the principal eigenvalue of $\left(-\Delta_{p}, W_{0}^{1, p}(\Omega)\right)$. We

\section{* Corresponding author.}

This is an Open Access article published by World Scientific Publishing Company. It is distributed under the terms of the Creative Commons Attribution 4.0 (CC-BY) License. Further distribution of this work is permitted, provided the original work is properly cited. 
look for positive solutions and prove a bifurcation-type theorem describing in an exact way the dependence of the set of positive solutions on the parameter $\lambda>0$.

Keywords: Parametric singular term; $(p-1)$-linear perturbation; uniform nonresonance; nonlinear regularity theory; truncation; strong comparison principle; bifurcation-type theorem.

Mathematics Subject Classification: 35J92, 35P30

\section{Introduction}

Let $\Omega \subseteq \mathbb{R}^{N}$ be a bounded domain with $C^{2}$-boundary $\partial \Omega$. In this paper, we study the following nonlinear parametric singular Dirichlet problem:

$$
\left\{\begin{array}{c}
-\Delta_{p} u(z)=\lambda u(z)^{-\gamma}+f(z, u(z)) \text { in } \Omega, \\
\left.u\right|_{\partial \Omega}=0, \quad u>0, \lambda>0,0<\gamma<1 .
\end{array}\right\}
$$

In this problem, $\Delta_{p}$ denotes the $p$-Laplacian differential operator defined by

$$
\Delta_{p} u=\operatorname{div}\left(|D u|^{p-2} D u\right) \quad \text { for all } u \in W_{0}^{1, p}(\Omega), \quad 1<p<\infty .
$$

On the right-hand side of $P_{\lambda}$ (the reaction of the problem), we have a parametric singular term $u \mapsto \lambda u^{-\gamma}$ with $\lambda>0$ being the parameter and $0<\gamma<1$. Also, there is a Carathéodory perturbation $f(z, x)$ (that is, for all $x \in \mathbb{R}$ the mapping $z \mapsto f(z, x)$ is measurable and for almost all $z \in \Omega$ the mapping $x \mapsto f(z, x)$ is continuous). We assume that $f(z, \cdot)$ exhibits $(p-1)$-linear growth near $+\infty$.

We are looking for positive solutions of problem $P_{\lambda}$. Our aim is to describe in a precise way the dependence on the parameter $\lambda>0$ of the set of positive solutions.

We prove a bifurcation-type property, which is the main result of our paper. Concerning the hypotheses $H(f)$ on the perturbation $f(z, x)$ and the other notation used in the statement of the theorem, we refer to Sec. 2] The main result of the present paper is stated in the following theorem.

Theorem A. If hypotheses $H(f)$ hold, then there exists $\lambda^{*} \in(0,+\infty)$ such that

(a) for every $\lambda \in\left(0, \lambda^{*}\right)$, problem $P_{\lambda}$ has at least two positive solutions

$$
u_{\lambda}, \hat{u}_{\lambda} \in \operatorname{int} C_{+}, u_{\lambda} \neq \hat{u}_{\lambda}, u_{\lambda} \leq \hat{u}_{\lambda}
$$

(b) for $\lambda=\lambda^{*}$, problem $P_{\lambda}$ has at least one positive solution

$$
u_{\lambda}^{*} \in \operatorname{int} C_{+}
$$

(c) for $\lambda>\lambda^{*}$, problem $P_{\lambda}$ has no positive solutions.

In the past, singular problems were studied in the context of semilinear equations (that is, $p=2$ ). We mention the works of Coclite and Palmieri 2, Ghergu and Rădulescu [5], Hirano et al. [10, Lair and Shaker [11, Sun et al. [21]. A detailed bibliography and additional topics on the subject can be found in the book of Ghergu and Rădulescu [6]. For nonlinear equations driven by the $p$-Laplacian, we mention the works of Giacomoni et al. [7, Papageorgiou et al. [16, 17, Papageorgiou 
and Smyrlis [18, Perera and Zhang [19. Of the aforementioned papers, closest to our work here is that of Papageorgiou and Smyrlis [18, where the authors also deal with a parametric singular problem and prove a bifurcation-type result. In their problem, the perturbation $f(z, x)$ is $(p-1)$-superlinear in $x \in \mathbb{R}$ near $+\infty$. So, our present work complements the results of [18, by considering equations in which the reaction has the competing effects of a singular term and of a $(p-1)$-linear term.

Our approach uses variational tools together with suitable truncation and comparison techniques.

\section{Preliminaries and Hypotheses}

Let $X$ be a Banach space and $X^{*}$ its topological dual. By $\langle\cdot, \cdot\rangle$ we denote the duality brackets of the pair $\left(X^{*}, X\right)$. Given $\varphi \in C^{1}(X, \mathbb{R})$, we say that $\varphi$ satisfies the "Cerami condition" (the "C-condition" for short), if the following property holds:

$$
\begin{aligned}
& \text { "Every sequence }\left\{u_{n}\right\}_{n \geq 1} \subseteq X \text { such that } \\
& \left\{\varphi\left(u_{n}\right)\right\}_{n \geq 1} \subseteq \mathbb{R} \text { is bounded and }\left(1+\left\|u_{n}\right\|\right) \varphi^{\prime}\left(u_{n}\right) \rightarrow 0 \text { in } X^{*} \text { as } n \rightarrow \infty, \\
& \text { admits a strongly convergent subsequence." }
\end{aligned}
$$

Using this notion, we can state the "mountain pass theorem".

Theorem 1 (Mountain pass theorem). Assume that $\varphi \in C^{1}(X, \mathbb{R})$ satisfies the $C$-condition, $u_{0}, u_{1} \in X,\left\|u_{1}-u_{0}\right\|>\rho>0$,

$$
\max \left\{\varphi\left(u_{0}\right), \varphi\left(u_{1}\right)\right\}<\inf \left\{\varphi(u):\left\|u-u_{0}\right\|=\rho\right\}=m_{\rho}
$$

and $c=\inf _{\gamma \in \Gamma} \max _{0 \leq t \leq 1} \varphi(\gamma(t))$ with $\Gamma=\left\{\gamma \in C([0,1], X): \gamma(0)=u_{0}, \gamma(1)=\right.$ $\left.u_{1}\right\}$. Then $c \geq m_{\rho}$ and $c$ is a critical value of $\varphi$ (that is, we can find $\hat{u} \in X$ such that $\varphi^{\prime}(\hat{u})=0$ and $\left.\varphi(\hat{u})=c\right)$.

The analysis of problem $P_{\lambda}$ will involve the Sobolev space $W_{0}^{1, p}(\Omega)$ and the Banach space

$$
C_{0}^{1}(\bar{\Omega})=\left\{u \in C^{1}(\bar{\Omega}):\left.u\right|_{\partial \Omega}=0\right\} .
$$

We denote by $\|\cdot\|$ the norm of $W_{0}^{1, p}(\Omega)$. On account of the Poincaré inequality, we have

$$
\|u\|=\|D u\|_{p} \quad \text { for all } u \in W_{0}^{1, p}(\Omega) .
$$

The space $C_{0}^{1}(\bar{\Omega})$ is an ordered Banach space with positive (order) cone

$$
C_{+}=\left\{u \in C_{0}^{1}(\bar{\Omega}): u(z) \geq 0 \text { for all } z \in \bar{\Omega}\right\} .
$$

This cone has a nonempty interior given by

$$
\operatorname{int} C_{+}=\left\{u \in C_{+}: u(z)>0 \text { for all } z \in \Omega,\left.\frac{\partial u}{\partial n}\right|_{\partial \Omega}<0\right\} \text {. }
$$

Here, $n(\cdot)$ denotes the outward unit normal on $\partial \Omega$. 
Let $h_{1}, h_{2} \in L^{\infty}(\Omega)$. We write $h_{1} \prec h_{2}$, if for every compact $K \subseteq \Omega$, we can find $c_{K}>0$ such that $c_{K} \leq h_{2}(z)-h_{1}(z)$ for almost all $z \in K$. Note that, if $h_{1}, h_{2} \in C(\Omega)$ and $h_{1}(z)<h_{2}(z)$ for all $z \in \Omega$, then $h_{1} \prec h_{2}$.

The next strong comparison principle can be found in Papageorgiou and Smyrlis [18. Proposition 4] (see also Giacomoni et al. [7, Theorem 2.3]).

Proposition 2. If $\hat{\xi} \geq 0, h_{1}, h_{2} \in L^{\infty}(\Omega), h_{1} \prec h_{2}, u_{1} \in C_{+}$with $u_{1}(z)>0$ for all $z \in \Omega, u_{2} \in \operatorname{int} C_{+}$and

$$
\begin{aligned}
& -\Delta_{p} u_{1}(z)+\hat{\xi} u_{1}(z)^{p-1}-\lambda u_{1}(z)^{-\gamma}=h_{1}(z), \\
& -\Delta_{p} u_{2}(z)+\hat{\xi} u_{2}(z)^{p-1}-\lambda u_{2}(z)^{-\gamma}=h_{2}(z) \text { for almost all } z \in \Omega,
\end{aligned}
$$

then $u_{2}-u_{1} \in \operatorname{int} C_{+}$.

We denote by $A: W_{0}^{1, p}(\Omega) \rightarrow W^{-1, p^{\prime}}(\Omega)=W_{0}^{1, p}(\Omega)^{*}\left(\frac{1}{p}+\frac{1}{p^{\prime}}=1\right)$ the nonlinear map defined by

$$
\langle A(u), h\rangle=\int_{\Omega}|D u|^{p-2}(D u, D h)_{\mathbb{R}^{N}} d z \quad \text { for all } u, h \in W_{0}^{1, p}(\Omega) .
$$

This map has the following properties (see Motreanu et al. [15, p. 40]).

Proposition 3. The map $A: W_{0}^{1, p}(\Omega) \rightarrow W^{-1, p^{\prime}}(\Omega)$ is bounded (that is, A maps bounded sets to bounded sets), continuous, strictly monotone and of type $(S)_{+}$, that is, if $u_{n} \stackrel{w}{\rightarrow} u$ in $W_{0}^{1, p}(\Omega)$ and $\limsup _{n \rightarrow \infty}\left\langle A\left(u_{n}\right), u_{n}-u\right\rangle \leq 0$, then $u_{n} \rightarrow u$ in $W_{0}^{1, p}(\Omega)$.

Consider the following nonlinear eigenvalue problem:

$$
-\Delta_{p} u(z)=\hat{\lambda}|u(z)|^{p-2} u(z) \quad \text { in } \Omega,\left.u\right|_{\partial \Omega}=0 .
$$

We say that $\hat{\lambda} \in \mathbb{R}$ is an "eigenvalue" of $\left(-\Delta_{p}, W_{0}^{1, p}(\Omega)\right)$ if problem (11) admits a nontrivial solution $\hat{u} \in W_{0}^{1, p}(\Omega)$, known as an "eigenfunction" corresponding to $\hat{\lambda}$. The nonlinear regularity theory (see Gasinski and Papageorgiou [3, pp. 737-738]) implies that $\hat{u} \in C_{0}^{1}(\bar{\Omega})$. There is a smallest eigenvalue $\hat{\lambda}_{1}>0$ with the following properties:

- $\hat{\lambda}_{1}>0$ is isolated (that is, if $\hat{\sigma}(p)$ denotes the spectrum of $\left(-\Delta_{p}, W_{0}^{1, p}(\Omega)\right)$ then we can find $\epsilon>0$ such that $\left.\left(\hat{\lambda}_{1}, \hat{\lambda}_{1}+\epsilon\right) \cap \hat{\sigma}(p)=0\right)$;

- $\hat{\lambda}_{1}$ is simple (that is, if $\hat{u}, \hat{v} \in C_{0}^{1}(\bar{\Omega})$ are eigenfunctions corresponding to $\hat{\lambda}_{1}$, then $\hat{u}=\xi \hat{v}$ for some $\xi \in \mathbb{R} \backslash\{0\})$;

$$
\hat{\lambda}_{1}=\inf \left\{\frac{\|D u\|_{p}^{p}}{\|u\|_{p}^{p}}: u \in W_{0}^{1, p}(\Omega), u \neq 0\right\} .
$$

It follows from the above properties that the eigenfunctions corresponding to $\hat{\lambda}_{1}$ do not change sign. We denote by $\hat{u}_{1}$ the positive, $L^{p}$-normalized (that is, $\left\|\hat{u}_{1}\right\|_{p}=1$ ) eigenfunction corresponding to $\hat{\lambda}_{1}>0$. From the nonlinear maximum principle (see, for example, Gasinski and Papageorgiou [3, p. 738]), we have $\hat{u}_{1} \in \operatorname{int} C_{+}$. 
Any eigenfunction corresponding to an eigenvalue $\hat{\lambda} \neq \hat{\lambda}_{1}$, is nodal (that is, signchanging). More details about the spectrum of $\left(-\Delta_{p}, W_{0}^{1, p}(\Omega)\right)$ can be found in [3. 15].

We can also consider a weighted version of the eigenvalue problem (10). So, let $m \in L^{\infty}(\Omega), m(z) \geq 0$ for almost all $z \in \Omega, m \neq 0$. We consider the following nonlinear eigenvalue problem:

$$
-\Delta_{p} u(z)=\tilde{\lambda} m(z)|u(z)|^{p-2} u(z) \text { in } \Omega,\left.\quad u\right|_{\partial \Omega}=0 .
$$

This problem has the same properties as (11). So, there is a smallest eigenvalue $\tilde{\lambda}_{1}(m)>0$ which is isolated, simple and admits the following variational characterization:

$$
\tilde{\lambda}_{1}(m)=\inf \left\{\frac{\|D u\|_{p}^{p}}{\int_{\Omega} m(z)|u|^{p} d z}: u \in W_{0}^{1, p}(\Omega), u \neq 0\right\} .
$$

Also the eigenfunctions corresponding to $\tilde{\lambda}_{1}(m)$ have a fixed sign and we denote by $\tilde{u}_{1}(m)$ the positive, $L^{p}$-normalized eigenfunction. We have $\tilde{u}_{1}(m) \in \operatorname{int} C_{+}$. These properties lead to the following monotonicity property of the map $m \mapsto \tilde{\lambda}_{1}(m)$.

Proposition 4. If $m_{1}, m_{2} \in L^{\infty}(\Omega), 0 \leq m_{1}(z) \leq m_{2}(z)$ for almost all $z \in \Omega$ and both inequalities are strict on sets of positive measure, then $\tilde{\lambda}_{1}\left(m_{2}\right)<\tilde{\lambda}_{1}\left(m_{1}\right)$.

Given $x \in \mathbb{R}$, we set $x^{ \pm}=\max \{ \pm x, 0\}$. Then for $u \in W_{0}^{1, p}(\Omega)$, we set $u^{ \pm}(\cdot)=$ $u(\cdot)^{ \pm}$. We know that

$$
u^{ \pm} \in W_{0}^{1, p}(\Omega), \quad|u|=u^{+}+u^{-}, \quad u=u^{+}-u^{-} .
$$

If $g: \Omega \times \mathbb{R}$ is a measurable function (for example, a Carathéodory function) then by $N_{g}(\cdot)$ we denote the Nemytski map corresponding to $g(\cdot, \cdot)$ defined by

$$
N_{g}(u)(\cdot)=g(\cdot, u(\cdot)) \quad \text { for all } u \in W_{0}^{1, p}(\Omega) .
$$

Given $v, u \in W_{0}^{1, p}(\Omega)$ with $v \leq u$, we define the order interval $[v, u]$ by

$$
[v, u]=\left\{y \in W_{0}^{1, p}(\Omega): v(z) \leq y(z) \leq u(z) \text { for almost all } z \in \Omega\right\} .
$$

The hypotheses on the perturbation $f(z, x)$ are the following:

$H(f): f: \Omega \times \mathbb{R} \leftarrow \mathbb{R}$ is a Carathéodory function such that $f(z, 0)=0$ for almost all $z \in \Omega$ and

(i) for every $\rho>0$, there exists $a_{\rho} \in L^{\infty}(\Omega)$ such that

$$
|f(z, x)| \leq a_{\rho}(z) \quad \text { for almost all } z \in \Omega \text {, and all } 0 \leq x \leq \rho ;
$$

(ii) $\hat{\lambda}_{1}<\eta \leq \liminf _{x \rightarrow+\infty} \frac{f(z, x)}{x^{p-1}} \leq \lim \sup _{x \rightarrow+\infty} \frac{f(z, x)}{x^{p-1}} \leq \hat{\eta}$ uniformly for almost all $z \in \Omega$; 
(iii) there exists a function $w \in C^{1}(\bar{\Omega})$ such that

$$
\begin{aligned}
w(z) & \geq c_{0}>0 \quad \text { for all } z \in \bar{\Omega}, \quad \Delta_{p} w \in L^{\infty}(\Omega) \text { with } \Delta_{p} w(z) \\
& \leq 0 \text { for almost all } z \in \Omega,
\end{aligned}
$$

and for every compact $K \subseteq \Omega$ we can find $c_{K}>0$ such that

$$
w(z)^{-\gamma}+f(z, w(z)) \leq-c_{K}<0 \quad \text { for almost all } z \in K ;
$$

(iv) there exists $\delta_{0} \in\left(0, c_{0}\right)$ such that for every compact $K \subseteq \Omega$

$$
f(z, x) \geq \hat{c}_{K}>0 \quad \text { for almost all } z \in K, \text { and all } x \in\left(0, \delta_{0}\right]
$$

(v) for every $\rho>0$, there exists $\hat{\xi}_{\rho}>0$ such that for almost all $z \in \Omega$ the function

$$
x \mapsto f(z, x)+\hat{\xi}_{\rho} x^{p-1}
$$

is nondecreasing on $[0, \rho]$.

Remark 1. Since we are looking for positive solutions and all the above hypotheses concern the positive semiaxis $\mathbb{R}_{+}=[0,+\infty)$, we may assume without any loss of generality that

$$
f(z, x)=0 \quad \text { for almost all } z \in \Omega \text {, and all } x \leq 0 .
$$

Hypothesis $H(f)$ (iii) implies that asymptotically at $+\infty$ we have uniform nonresonance with respect to the principal eigenvalue $\hat{\lambda}_{1}>0$ of $\left(-\Delta_{p}, W_{0}^{1, p}(\Omega)\right)$. The resonant case was recently examined for nonparametric singular Dirichlet problems by Papageorgiou et al. [16].

Example 1. The following functions satisfy hypotheses $H(f)$. For the sake of simplicity we drop the $z$-dependence:

$$
\left.f(x)=\left\{\begin{array}{ll}
x^{\tau-1}-3 x^{\vartheta-1} & \text { if } 0 \leq x \leq 1 \\
\eta x^{p-1}-(\eta+2) x^{q-1} & \text { if } 1<x
\end{array}\right\} \text { (see (4) }\right)
$$

with $1<\tau<\vartheta, 1<q<p$ and $\eta>\hat{\lambda}_{1}$; and

$$
f(x)= \begin{cases}2 \sin (2 \pi x) & \text { if } 0 \leq x \leq 1 \\ \eta\left(x^{p-1}-x^{q-1}\right) & \text { if } 1<x\end{cases}
$$

with $\eta>\hat{\lambda}_{1}, 1<q<p$. 


\section{A Purely Singular Problem}

In this section we deal with the following purely singular parametric problem:

$$
\left\{\begin{array}{c}
-\Delta_{p} u(z)=\lambda u(z)^{-\gamma} \text { in } \Omega \\
\left.u\right|_{\partial \Omega}=0, u>0, \lambda>0,0<\gamma<1
\end{array}\right\}
$$

The next proposition establishes the existence and $\lambda$-dependence of the positive solutions for problem $A u_{\lambda}$.

Proposition 5. For every $\lambda>0$ problem $A u_{\lambda}$ admits a unique solution $\tilde{u}_{\lambda} \in$ $\operatorname{int} C_{+}$, the map $\lambda \mapsto \tilde{u}_{\lambda}$ is nondecreasing from $(0, \infty)$ into $C_{0}^{1}(\bar{\Omega})$ (that is, if $0<$ $\vartheta<\lambda$, then $\left.\tilde{u}_{\vartheta} \leq \tilde{u}_{\lambda}\right)$ and $\left\|\tilde{u}_{\lambda}\right\|_{C_{0}^{1}(\bar{\Omega})} \rightarrow 0$ as $\lambda \rightarrow 0^{+}$.

Proof. The existence of a unique solution $\tilde{u}_{\lambda} \in \operatorname{int} C_{+}$follows from Proposition 5 of Papageorgiou and Smyrlis [18].

Let $0<\vartheta<\lambda$ and let $\tilde{u}_{\vartheta}, \tilde{u}_{\lambda} \in \operatorname{int} C_{+}$be the corresponding unique solutions of problem $A u_{\lambda}$. Evidently, $\tilde{u}_{\vartheta}^{1 / p^{\prime}} \in C_{+}\left(\frac{1}{p}+\frac{1}{p^{\prime}}=1\right)$ and so by Proposition 2.1 of Marano and Papageorgiou [14, we can find $c_{1}>0$ such that

$$
\begin{aligned}
& \hat{u}_{1}^{1 / p^{\prime}} \leq c_{1}^{1 / p^{\prime}} \tilde{u}_{\vartheta}, \\
\Rightarrow & \tilde{u}_{\vartheta}^{-\gamma} \leq c_{2} \hat{u}_{1}^{-\gamma / p^{\prime}} \quad \text { for some } c_{2}>0 .
\end{aligned}
$$

The Lemma of Lazer and McKenna [12, p. 726], implies that $\hat{u}_{1}^{-\gamma / p^{\prime}} \in L^{p^{\prime}}(\Omega)$. Therefore, $\tilde{u}_{\vartheta}^{-\gamma} \in L^{p^{\prime}}(\Omega)$. We introduce the Carathéodory function $g_{\lambda}(z, x)$ defined by

$$
g_{\lambda}(z, x)= \begin{cases}\lambda \tilde{u}_{\vartheta}^{-\gamma} & \text { if } x \leq \tilde{u}_{\vartheta}(z) \\ \lambda x^{-\gamma} & \text { if } \tilde{u}_{\vartheta}(z)<x .\end{cases}
$$

We set $G_{\lambda}(z, x)=\int_{0}^{x} g_{\lambda}(z, s) d s$ and consider the functional $\hat{\psi}_{\lambda}: W_{0}^{1, p}(\Omega) \rightarrow \mathbb{R}$ defined by

$$
\hat{\psi}_{\lambda}(u)=\frac{1}{p}\|D u\|_{p}^{p}-\int_{\Omega} G_{\lambda}(z, u) d z \quad \text { for all } u \in W_{0}^{1, p}(\Omega) .
$$

Proposition 3 of Papageorgiou and Smyrlis 18 implies that $\hat{\psi}_{\lambda} \in C^{1}\left(W_{0}^{1, p}(\Omega)\right)$. From (5) and since $\tilde{u}_{\vartheta}^{-\gamma} \in L^{p^{\prime}}(\Omega)$ it follows that $\hat{\psi}_{\lambda}(\cdot)$ is coercive. Also, via the Sobolev embedding theorem, we see that $\hat{\psi}_{\lambda}(\cdot)$ is sequentially weakly lower semicontinuous. So, by the Weierstrass-Tonelli theorem, we can find $\bar{u}_{\lambda} \in W_{0}^{1, p}(\Omega)$ such that

$$
\begin{aligned}
& \hat{\psi}_{\lambda}\left(\bar{u}_{\lambda}\right)=\inf \left\{\hat{\psi}_{\lambda}(u): u \in W_{0}^{1, p}(\Omega)\right\} \\
\Rightarrow & \hat{\psi}_{\lambda}^{\prime}\left(\bar{u}_{\lambda}\right)=0 \\
\Rightarrow & \left\langle A\left(\bar{u}_{\lambda}\right), h\right\rangle=\int_{\Omega} g_{\lambda}\left(z, \bar{u}_{\lambda}\right) h d z \quad \text { for all } h \in W_{0}^{1, p}(\Omega) .
\end{aligned}
$$


In (3) we choose $h=\left(\tilde{u}_{\vartheta}-\bar{u}_{\lambda}\right)^{+} \in W_{0}^{1, p}(\Omega)$. We have

$$
\begin{aligned}
\left\langle A\left(\bar{u}_{\lambda}\right),\left(\tilde{u}_{\vartheta}-\bar{u}_{\lambda}\right)^{+}\right\rangle & =\int_{\Omega} \lambda \tilde{u}_{\vartheta}^{-\gamma}\left(\tilde{u}_{\vartheta}-\bar{u}_{\lambda}\right)^{+} d z(\text { see (5) }) \\
& \geq \int_{\Omega} \vartheta \tilde{u}_{\vartheta}^{-\gamma}\left(\tilde{u}_{\vartheta}-\bar{u}_{\lambda}\right) d z(\text { since } \vartheta<\lambda) \\
& =\left\langle A\left(\tilde{u}_{\vartheta}\right),\left(\tilde{u}_{\vartheta}-\bar{u}_{\lambda}\right)^{+}\right\rangle, \\
\Rightarrow \tilde{u}_{\vartheta} & \leq \bar{u}_{\lambda} .
\end{aligned}
$$

From (5), (6), (7), we have

$$
\begin{aligned}
& -\Delta_{p} \bar{u}_{\lambda}(z)=\lambda \bar{u}_{\lambda}(z)^{-\gamma} \text { for almost all } z \in \Omega,\left.\bar{u}_{\lambda}\right|_{\partial \Omega}=0, \\
\Rightarrow & \bar{u}_{\lambda}=\tilde{u}_{\lambda} \\
\Rightarrow & \left.\tilde{u}_{\vartheta} \leq \tilde{u}_{\lambda} \text { (see (7D) }\right) .
\end{aligned}
$$

Therefore, the map $\lambda \mapsto \tilde{u}_{\lambda}$ is nondecreasing from $(0,+\infty)$ into $C_{0}^{1}(\bar{\Omega})$.

We have

$$
\left\langle A\left(\tilde{u}_{\lambda}\right), h\right\rangle=\int_{\Omega} \lambda \tilde{u}_{\lambda}^{-\gamma} h d z \quad \text { for all } h \in W_{0}^{1, p}(\Omega) .
$$

Choosing $h=\tilde{u}_{\lambda} \in W_{0}^{1, p}(\Omega)$, we obtain

$$
\left\|D \tilde{u}_{\lambda}\right\|_{p}^{p}=\lambda \int_{\Omega} \tilde{u}_{\lambda}^{1-\gamma} d z \leq \lambda c_{3}\left\|\tilde{u}_{\lambda}\right\|_{p} \quad \text { for some } c_{3}>0
$$

(see Theorem 13.17 of Hewitt and Stromberg [9, p. 196]),

$$
\Rightarrow\left\{\tilde{u}_{\lambda}\right\}_{\lambda \in(0,1]} \subseteq W_{0}^{1, p}(\Omega) \text { is bounded and }\left\|\tilde{u}_{\lambda}\right\| \rightarrow 0 \text { as } \lambda \rightarrow 0^{+} .
$$

As in the first part of the proof, using Proposition 2.1 of Marano and Papageorgiou [14, we show that $\tilde{u}_{\lambda}^{-\gamma} \in L^{r}(\Omega)$ for $r>N$. Then Proposition 1.3 of Guedda and Véron [8] implies that

$$
\tilde{u}_{\lambda} \in L^{\infty}(\Omega) \text { and }\left\|\tilde{u}_{\lambda}\right\|_{\infty} \leq c_{4} \text { for some } c_{4}>0 \text {, and all } 0<\lambda \leq 1 .
$$

Let $k_{\lambda}=\lambda \tilde{u}_{\lambda}^{-\gamma} \in L^{r}(\Omega), \lambda \in(0,1]$ and consider the following linear Dirichlet problem:

$$
-\Delta v(z)=k_{\lambda}(z) \text { in } \Omega,\left.\quad v\right|_{\partial \Omega}=0,0<\lambda \leq 1 .
$$

Standard existence and regularity theory (see, for example, Struwe [20, p. 218]), implies that problem (10) has a unique solution $v_{\lambda}(\cdot)$ such that

$$
v_{\lambda} \in W^{2, r}(\Omega) \subseteq C_{0}^{1, \alpha}(\bar{\Omega})=C^{1, \alpha}(\bar{\Omega}) \cap C_{0}^{1}(\bar{\Omega}), \quad\left\|v_{\lambda}\right\|_{C_{0}^{1, \alpha}(\bar{\Omega})} \leq c_{5}
$$

for some $c_{5}>0$, all $\lambda \in(0,1]$, and with $\alpha=1-\frac{N}{r} \in(0,1)$ (recall that $\left.r>N\right)$. Let $\beta_{\lambda}(z)=D v_{\lambda}(z)$. Then $\beta_{\lambda} \in C^{0, \alpha}(\bar{\Omega})$ for every $\lambda \in(0,1]$. We have

$-\operatorname{div}\left[\left|D \tilde{u}_{\lambda}\right|^{p-2} D \tilde{u}_{\lambda}-\beta_{\lambda}\right]=0$ in $\Omega,\left.\quad \tilde{u}_{\lambda}\right|_{\partial \Omega}=0$ (since $\tilde{u}_{\lambda}$ solves $\left.A u_{\lambda}\right)$. 
Then Theorem 1 of Lieberman [13] (see also Corollary 1.1 of Guedda and Véron [8]) and (99), imply that we can find $s \in(0,1)$ and $c_{6}>0$ such that

$$
\tilde{u}_{\lambda} \in C_{0}^{1, s}(\bar{\Omega}) \cap \operatorname{int} C_{+}, \quad\left\|\tilde{u}_{\lambda}\right\|_{C_{0}^{1, s}(\bar{\Omega})} \leq c_{6} \quad \text { for all } \lambda \in(0,1] .
$$

Finally, the compact embedding of $C_{0}^{1, s}(\bar{\Omega})$ into $C_{0}^{1}(\bar{\Omega})$ and (8) imply that

$$
\left\|\tilde{u}_{\lambda}\right\|_{C_{0}^{1}(\bar{\Omega})} \rightarrow 0 \text { as } \lambda \rightarrow 0^{+} .
$$

This completes the proof.

\section{Bifurcation-Type Theorem}

Let

$$
\begin{aligned}
\mathcal{L} & =\{\lambda>0 \text { : problem } P \text { admits a positive solution }\} \\
S_{\lambda} & =\text { the set of positive solutions for problem } P_{\lambda} .
\end{aligned}
$$

Proposition 6. If hypotheses $H(f)$ hold, then $\mathcal{L} \neq \emptyset$.

Proof. Using Proposition [5, we can find $\lambda_{0} \in(0,1]$ such that

$$
\tilde{u}_{\lambda}(z) \in\left(0, \delta_{0}\right] \text { for all } z \in \Omega, \quad \text { all } \lambda \in\left(0, \lambda_{0}\right] \text {. }
$$

Here, $\delta_{0}>0$ is as postulated by hypothesis $H(f)(i v)$.

We fix $\lambda \in\left(0, \lambda_{0}\right]$ and we consider the following truncation of the reaction in problem $\left.P_{\lambda}\right)$ :

$$
\hat{k}_{\lambda}(z, x)= \begin{cases}\lambda \hat{u}_{\lambda}(z)^{-\gamma}+f\left(z, \tilde{u}_{\lambda}(z)\right) & \text { if } x<\tilde{u}_{\lambda}(z), \\ \lambda x^{-\gamma}+f(z, x) & \text { if } \tilde{u}_{\lambda} \leq x \leq w(z), \\ \lambda w(z)^{-\gamma}+f(z, w(z)) & \text { if } w(z)<x .\end{cases}
$$

(recall that $\delta_{0}<c_{0} \leq w(z)$ for all $z \in \bar{\Omega}$ ). This is a Carathéodory function. We set $\hat{K}_{\lambda}(z, x)=\int_{0}^{x} \hat{k}_{\lambda}(z, s) d s$ and consider the function $\hat{\varphi}_{\lambda}: W_{0}^{1, p}(\Omega) \rightarrow \mathbb{R}$ defined by

$$
\hat{\varphi}_{\lambda}(u)=\frac{1}{p}\|D u\|_{p}^{p}-\int_{\Omega} \hat{K}_{\lambda}(z, u) d z \quad \text { for all } u \in W_{0}^{1, p}(\Omega) .
$$

As before, we have $\hat{\varphi}_{\lambda} \in C^{1}\left(W_{0}^{1, p}(\Omega)\right)$. Also, it follows from (12) that

$$
\hat{\varphi}(\cdot) \text { is coercive. }
$$

In addition, we have that

$$
\hat{\varphi}_{\lambda}(\cdot) \text { is sequentially lower semicontinuous. }
$$

Therefore, we can find $\hat{u}_{\lambda} \in W_{0}^{1, p}(\Omega)$ such that

$$
\begin{aligned}
& \hat{\varphi}_{\lambda}\left(\hat{u}_{\lambda}\right)=\inf \left[\hat{\varphi}_{\lambda}(u): u \in W_{0}^{1, p}(\Omega)\right], \\
\Rightarrow & \hat{\varphi}_{\lambda}^{\prime}\left(\hat{u}_{\lambda}\right)=0, \\
\Rightarrow & \left\langle A\left(\hat{u}_{\lambda}\right), h\right\rangle=\int_{\Omega} \hat{k}_{\lambda}\left(z, \hat{u}_{\lambda}\right) h d z \quad \text { for all } h \in W_{0}^{1, p}(\Omega) .
\end{aligned}
$$


In (13) we choose $h=\left(\tilde{u}_{\lambda}-\hat{u}_{\lambda}\right)^{+} \in W_{0}^{1, p}(\Omega)$. Then

$$
\begin{aligned}
\left\langle A\left(\hat{u}_{\lambda}\right),\left(\tilde{u}_{\lambda}-\hat{u}_{\lambda}\right)^{+}\right\rangle= & \int_{\Omega}\left[\lambda \tilde{u}_{\lambda}^{-\gamma}+f\left(z, \tilde{u}_{\lambda}\right)\right]\left(\tilde{u}_{\lambda}-\hat{u}_{\lambda}\right)^{+} d z(\text { see (12) }) \\
\geq & \int_{\Omega} \lambda \tilde{u}_{\lambda}^{-\gamma}\left(\tilde{u}_{\lambda}-\hat{u}_{\lambda}\right)^{+} d z \\
& (\text { see (11) and hypothesis } H(f)(\text { iv })) \\
= & \left\langle A\left(\tilde{u}_{\lambda}\right),\left(\tilde{u}_{\lambda}-\hat{u}_{\lambda}\right)^{+}\right\rangle(\text {see Proposition [5) } \\
\Rightarrow \tilde{u}_{\lambda} \leq & \hat{u}_{\lambda} .
\end{aligned}
$$

Next, we choose $h=\left(\hat{u}_{\lambda}-w\right)^{+} \in W_{0}^{1, p}(\Omega)$ in (13). Then

$$
\begin{aligned}
\left\langle A\left(\hat{u}_{\lambda}\right),\left(\hat{u}_{\lambda}-w\right)^{+}\right\rangle & =\int_{\Omega}\left[\lambda w^{-\gamma}+f(z, w)\right]\left(\hat{u}_{\lambda}-w\right)^{+} d z(\text { see (12) }) \\
& \leq\left\langle A(w),\left(\hat{u}_{\lambda}-w\right)^{+}\right\rangle
\end{aligned}
$$

(see hypothesis $H(f)($ iii) and use the nonlinear Green identity, see [3, p. 211])

$$
\Rightarrow \tilde{u}_{\lambda} \leq w
$$

So, we have proved that

$$
\hat{u}_{\lambda} \in\left[\tilde{u}_{\lambda}, w\right] .
$$

Using (14) and (12), Eq. (13) becomes

$$
\begin{aligned}
\left\langle A\left(\hat{u}_{\lambda}\right), h\right\rangle & =\int_{\Omega}\left[\lambda \hat{u}_{\lambda}^{-\gamma}+f\left(z, \hat{u}_{\lambda}\right)\right] h d z \quad \text { for all } h \in W_{0}^{1, p}(\Omega), \\
\Rightarrow-\Delta_{p} \hat{u}_{\lambda}(z) & =\lambda \hat{u}_{\lambda}(z)^{-\gamma}+f\left(z, \hat{u}_{\lambda}(z)\right) \quad \text { for almost all } z \in \Omega,\left.\hat{u}_{\lambda}\right|_{\partial \Omega}=0 .
\end{aligned}
$$

From (14), (15) and Theorem 1 of Lieberman [13], we infer that

$$
\begin{aligned}
& \hat{u}_{\lambda} \in\left[\tilde{u}_{\lambda}, w\right] \cap \operatorname{int} C_{+}, \\
\Rightarrow & \lambda \in \mathcal{L}, \hat{u}_{\lambda} \in S_{\lambda} .
\end{aligned}
$$

This completes the proof.

A byproduct of the above proof is the following corollary.

Corollary 7. If hypotheses $H(f)$ hold, then $S_{\lambda} \subseteq \operatorname{int} C_{+}$for all $\lambda>0$.

The next proposition shows that $\mathcal{L}$ is an interval.

Proposition 8. If hypotheses $H(f)$ hold, $\lambda \in \mathcal{L}$ and $\vartheta \in(0, \lambda)$, then $\vartheta \in \mathcal{L}$. 
Proof. Since $\lambda \in \mathcal{L}$, we can find $u_{\lambda} \in S_{\lambda} \subseteq \operatorname{int} C_{+}$. Proposition 5 implies that we can find $\tau \in\left[0, \lambda_{0}\right]$ (see (11) ) such that

$$
\tau<\vartheta \quad \text { and } \quad \tilde{u}_{\tau} \leq u_{\lambda}
$$

We introduce the Carathéodory function $e(z, x)$ defined by

$$
e_{\vartheta}(z, x)= \begin{cases}\vartheta \tilde{u}_{\tau}(z)^{-\gamma}+f\left(z, \tilde{u}_{\tau}(z)\right) & \text { if } x<\tilde{u}_{\tau}(z), \\ \vartheta x^{-\gamma}+f(z, x) & \text { if } \tilde{u}_{\tau}(z) \leq x \leq u_{\lambda}(z), \\ \vartheta u_{\lambda}(z)^{-\gamma}+f\left(z, u_{\lambda}(z)\right) & \text { if } u_{\lambda}(z)<x .\end{cases}
$$

We set $E_{\vartheta}(z, x)=\int_{0}^{x} e_{\vartheta}(z, s) d s$ and consider the functional $\hat{\psi}_{\vartheta}: W_{0}^{1, p}(\Omega) \rightarrow \mathbb{R}$ defined by

$$
\hat{\psi}_{\vartheta}(u)=\frac{1}{p}\|D u\|_{p}^{p}-\int_{\Omega} E_{\vartheta}(z, u) d z \quad \text { for all } u \in W_{0}^{1, p}(\Omega) .
$$

We know that $\hat{\psi}_{\vartheta} \in C^{1}\left(W_{0}^{1, p}(\Omega)\right)$. Moreover, $\hat{\psi}_{\vartheta}$ is coercive (see (16)) and sequentially weakly lower semicontinuous. So, we can find $u_{\vartheta} \in W_{0}^{1, p}(\Omega)$ such that

$$
\begin{aligned}
& \hat{\psi}_{\vartheta}\left(u_{\vartheta}\right)=\inf \left\{\hat{\psi}_{\vartheta}(u): u \in W_{0}^{1, p}(\Omega)\right\} \\
\Rightarrow & \hat{\psi}_{\vartheta}^{\prime}\left(u_{\vartheta}\right)=0, \\
\Rightarrow & \left\langle A\left(u_{\vartheta}\right), h\right\rangle=\int_{\Omega} e_{\vartheta}\left(z, u_{\vartheta}\right) h d z \quad \text { for all } h \in W_{0}^{1, p}(\Omega) .
\end{aligned}
$$

In (17) we first choose $h=\left(\tilde{u}_{\tau}-u_{\vartheta}\right)^{+} \in W_{0}^{1, p}(\Omega)$. Then

$$
\begin{aligned}
\left\langle A\left(u_{\vartheta}\right),\left(\tilde{u}_{\tau}-u_{\vartheta}\right)^{+}\right\rangle= & \int_{\Omega}\left[\vartheta \tilde{u}_{\tau}^{-\gamma}+f\left(z, \tilde{u}_{\tau}\right)\right]\left(\tilde{u}_{\tau}-u_{\vartheta}\right)^{+} d z(\text { see (16) }) \\
\geq & \int_{\Omega} \vartheta \tilde{u}_{\tau}^{-\gamma}\left(\tilde{u}_{\tau}-u_{\vartheta}\right)^{+} d z \\
& \left(\text { since } \tau \leq \lambda_{0}, \text { see (11) and hypothesis } H(f)(\mathrm{iv})\right) \\
\geq & \int_{\Omega} \tau \tilde{u}_{\tau}^{-\gamma}\left(\tilde{u}_{\tau}-u_{\vartheta}\right)^{+} d z(\text { recall that } \tau<\vartheta) \\
= & \left\langle A\left(u_{\tau}\right),\left(\tilde{u}_{\tau}-u_{\vartheta}\right)^{+}\right\rangle(\text {see Proposition } 5), \\
\Rightarrow \tilde{u}_{\tau} \leq & u_{\vartheta} .
\end{aligned}
$$

Next, in (17) we choose $h=\left(u_{\vartheta}-u_{\lambda}\right)^{+} \in W_{0}^{1, p}(\Omega)$. Then

$$
\begin{aligned}
\left\langle A\left(u_{\vartheta}\right),\left(u_{\vartheta}-u_{\lambda}\right)^{+}\right\rangle & =\int_{\Omega}\left[\vartheta u_{\lambda}^{-\gamma}+f\left(z, u_{\lambda}\right)\right]\left(u_{\vartheta}-u_{\lambda}\right)^{+} d z(\text { see (16) }) \\
& \leq \int_{\Omega}\left[\lambda u_{\lambda}^{-\gamma}+f\left(z, u_{\lambda}\right)\right]\left(u_{\vartheta}-u_{\lambda}\right)^{+} d z(\text { since } \vartheta<\lambda) \\
& =\left\langle A\left(u_{\lambda}\right),\left(u_{\vartheta}-u_{\lambda}\right)^{+}\right\rangle\left(\text {since } u_{\lambda} \in S_{\lambda}\right) \\
\Rightarrow u_{\vartheta} & \leq u_{\lambda} .
\end{aligned}
$$


So, we have proved that

$$
u_{\vartheta} \in\left[\tilde{u}_{\tau}, u_{\lambda}\right] .
$$

It follows from (16), (17) and (18) that

$$
\vartheta \in \mathcal{L} \quad \text { and } \quad u_{\vartheta} \in S_{\vartheta} \subseteq \operatorname{int} C_{+} .
$$

The proof is now complete.

An interesting byproduct of the above proof is the following result.

Corollary 9. If hypotheses $H(f)$ hold, $\lambda \in \mathcal{L}, u_{\lambda} \in S_{\lambda} \subseteq \operatorname{int} C_{+}$, and $\vartheta<\lambda$, then $\vartheta \in \mathcal{L}$ and we can find $u_{\vartheta} \in S_{\vartheta} \subseteq \operatorname{int} C_{+}$such that $u_{\vartheta} \leq u_{\lambda}$.

In fact, we can improve the above result as follows.

Proposition 10. If hypotheses $H(f)$ hold, $\lambda \in \mathcal{L}, u_{\lambda} \in S_{\lambda} \subseteq \operatorname{int} C_{+}$, and $\vartheta<\lambda$, then $\vartheta \in \mathcal{L}$ and we can find $u_{\vartheta} \in S_{\vartheta} \subseteq \operatorname{int} C_{+}$such that $u_{\lambda}-u_{\vartheta} \in \operatorname{int} C_{+}$.

Proof. From Corollary 9 we know that $\vartheta \in \mathcal{L}$ and we can find $u_{\vartheta} \in S_{\vartheta} \subseteq \operatorname{int} C_{+}$ such that

$$
u_{\vartheta} \leq u_{\lambda}
$$

Let $\rho=\left\|u_{\lambda}\right\|_{\infty}$ and let $\hat{\xi}_{\rho}>0$ be as postulated by hypothesis $H(f)(v)$. Then

$$
\begin{aligned}
& -\Delta_{p} u_{\vartheta}+\hat{\xi}_{p} u_{\vartheta}^{p-1}-\lambda u_{\vartheta}^{-\gamma} \\
& \quad=-(\lambda-\vartheta) u_{\vartheta}^{-\gamma}+f\left(z, u_{\vartheta}\right)+\hat{\xi}_{\rho} u_{\vartheta}^{p-1} \\
& \quad \leq f\left(z, u_{\lambda}\right)+\hat{\xi}_{\rho} u_{\lambda}^{p-1}(\text { recall that } \vartheta<\lambda \text { and see (19) and hypothesis } H(f)(\mathrm{v})) \\
& \left.=-\Delta_{p} u_{\lambda}+\hat{\xi}_{\rho} u_{\lambda}^{p-1}-\lambda u_{\lambda}^{-\gamma} \text { (since } u_{\lambda} \in S_{\lambda}\right) .
\end{aligned}
$$

We set

$$
\begin{aligned}
& h_{1}(z)=f\left(z, u_{\vartheta}(z)\right)+\hat{\xi}_{\rho} u_{\vartheta}(z)^{p-1}-(\lambda-\vartheta) u_{\vartheta}(z)^{-\gamma} \\
& h_{2}(z)=f\left(z, u_{\lambda}(z)\right)+\hat{\xi}_{\rho} u_{\lambda}(z)^{p-1} .
\end{aligned}
$$

We have

$$
h_{2}(z)-h_{1}(z) \geq(\lambda-\vartheta) u_{\vartheta}(z)^{-\gamma} \geq(\lambda-\vartheta) \rho^{-\gamma} \text { for almost all } z \in \Omega
$$

(see (19) and hypotheses $H(f)(\mathrm{v})$ ).

We can apply Proposition 2 and conclude that

$$
u_{\lambda}-u_{\vartheta} \in \operatorname{int} C_{+} .
$$

The proof is now complete.

Denote $\lambda^{*}=\sup \mathcal{L}$.

Proposition 11. If hypotheses $h(f)$ hold, then $\lambda^{*}<+\infty$. 
Proof. Let $\epsilon>0$ be such that $\hat{\lambda}_{1}+\epsilon<\eta$ (see hypothesis $H(f)($ ii $)$ ). We can find $M>0$ such that

$$
f(z, x) \geq\left[\hat{\lambda}_{1}+\epsilon\right] x^{p-1} \quad \text { for almost all } z \in \Omega, \text { and all } x \geq M .
$$

Also, hypothesis $H(f)(\mathrm{i})$ implies that we can find large enough $\tilde{\lambda}>0$ such that $\tilde{\lambda} M^{-\gamma}+f(z, x) \geq\left[\hat{\lambda}_{1}+\epsilon\right] M^{p-1}$ for almost all $z \in \Omega$, and all $0 \leq x \leq M$.

It follows from (20) and (21) that

$\tilde{\lambda} x^{-\gamma}+f(z, x) \geq\left[\hat{\lambda}_{1}+\epsilon\right] x^{p-1} \quad$ for almost all $z \in \Omega$, and all $x \geq 0$.

Let $\lambda>\tilde{\lambda}$ and suppose that $\lambda \in \mathcal{L}$. Then we can find $u_{\lambda} \in S_{\lambda} \subseteq \operatorname{int} C_{+}$. We have

$$
\begin{aligned}
-\Delta_{p} u_{\lambda} & =\lambda u_{\lambda}^{-\gamma}+f\left(z, u_{\lambda}\right)>\tilde{\lambda} u_{\lambda}^{-\gamma}+f\left(z, u_{\lambda}\right) \\
& \geq\left[\hat{\lambda}_{1}+\epsilon\right] u_{\lambda}^{p-1} \quad \text { for a.a. } z \in \Omega(\text { see (22) }) .
\end{aligned}
$$

Since $u_{\lambda} \in \operatorname{int} C_{+}$, we can find $t \in(0,1)$ so small that

$$
\hat{y}_{1}=t \hat{u}_{1} \leq u_{\lambda}
$$

(see Proposition 2.1 of Marano and Papageorgiou [14]). We have

$$
-\Delta_{p} \hat{y}_{1}=\hat{\lambda}_{1} \hat{y}_{1}^{p-1}<\left[\hat{\lambda}_{1}+\epsilon\right] \hat{y}_{1}^{p-1} \text { for almost all } z \in \Omega .
$$

Using (24), we can define the Carathéodory function $\beta(z, x)$ as follows:

$$
\beta(z, x)= \begin{cases}{\left[\hat{\lambda}_{1}+\epsilon\right] \hat{y}_{1}(z)^{p-1}} & \text { if } x<\hat{y}_{1}(z) \\ {\left[\hat{\lambda}_{1}+\epsilon\right] x^{p-1}} & \text { if } \hat{y}_{1}(z) \leq x \leq u_{\lambda}(z) \\ {\left[\hat{\lambda}_{1}+\epsilon\right] u_{\lambda}(z)^{p-1}} & \text { if } u_{\lambda}(z)<x\end{cases}
$$

We set $B(z, x)=\int_{0}^{x} \beta(z, s) d s$ and consider the $C^{1}$-functional $\sigma: W_{0}^{1, p}(\Omega) \rightarrow \mathbb{R}$ defined by

$$
\sigma(u)=\frac{1}{p}\|D u\|_{p}^{p}-\int_{\Omega} B(z, u) d z \quad \text { for all } u \in W_{0}^{1, p}(\Omega) .
$$

From (26) it is clear that $\sigma(\cdot)$ is coercive. Also, it is sequentially weakly lower semicontinuous. So, we can find $\bar{u} \in W_{0}^{1, p}(\Omega)$ such that

$$
\begin{aligned}
& \sigma(\bar{u})=\inf \left\{\sigma(u): u \in W_{0}^{1, p}(\Omega)\right\}, \\
\Rightarrow & \sigma^{\prime}(\bar{u})=0, \\
\Rightarrow & \langle A(\bar{u}), h\rangle=\int_{\Omega} \beta(z, \bar{u}) h d z \quad \text { for all } h \in W_{0}^{1, p}(\Omega) .
\end{aligned}
$$


In (27) we first choose $h=\left(\hat{y}_{1}-\bar{u}\right)^{+} \in W_{0}^{1, p}(\Omega)$. Then

$$
\begin{aligned}
\left\langle A(\bar{u}),\left(\hat{y}_{1}-\bar{u}\right)^{+}\right\rangle & =\int_{\Omega}\left[\hat{\lambda}_{1}+\epsilon\right] \hat{y}_{1}^{p-1}\left(\hat{y}_{1}-\bar{u}\right)^{+} d z(\text { see }(26)) \\
& \geq\left\langle A\left(\hat{y}_{1}\right),\left(\hat{y}_{1}-\hat{u}\right)^{+}\right\rangle(\operatorname{see}(\underline{25})) \\
\Rightarrow \hat{y}_{1} & \leq \bar{u} .
\end{aligned}
$$

Also, in (27) we choose $h=\left(\bar{u}-u_{\lambda}\right)^{+} \in W_{0}^{1, p}(\Omega)$. Then

$$
\begin{aligned}
\left\langle A(\bar{u}),\left(\bar{u}-u_{\lambda}\right)^{+}\right\rangle & =\int_{\Omega}\left[\hat{\lambda}_{1}+\epsilon\right] u_{\lambda}^{p-1}\left(\bar{u}-u_{\lambda}\right)^{+} d z(\text { see (26) }) \\
& \leq\left\langle A\left(u_{\lambda}\right),\left(\bar{u}-u_{\lambda}\right)^{+}\right\rangle(\text {see }(23)) \\
\Rightarrow \bar{u} & \leq u_{\lambda} .
\end{aligned}
$$

So, we have proved that

$$
\bar{u} \in\left[\hat{y}_{1}, u_{\lambda}\right] .
$$

It follows from (26), (27) and (28) that

$$
\begin{aligned}
& -\Delta_{p} \bar{u}(z)=\left[\hat{\lambda}_{1}+\epsilon\right] \bar{u}(z)^{p-1} \quad \text { for almost all } z \in \Omega,\left.\bar{u}\right|_{\partial \Omega}=0, \\
\Rightarrow & \left.\bar{u} \in C_{0}^{1}(\bar{\Omega}) \text { must be nodal, a contradiction (see (28) }\right) .
\end{aligned}
$$

Therefore, we have $\lambda^{*} \leq \tilde{\lambda}<+\infty$.

Next, we show that the critical parameter $\lambda^{*}>0$ is admissible.

Proposition 12. If hypotheses $H(f)$ hold, then $\lambda^{*} \in \mathcal{L}$.

Proof. Let $\left\{\lambda_{n}\right\}_{n \geq 1} \subseteq\left(0, \lambda^{*}\right)$ and assume that $\lambda_{n} \rightarrow\left(\lambda^{*}\right)^{-}$as $n \rightarrow \infty$. We can find $u_{n}=u_{\lambda_{n}} \in S_{\lambda_{n}} \subseteq \operatorname{int} C_{+}$for all $n \in \mathbb{N}$. Then

$$
\left\langle A\left(u_{n}\right), h\right\rangle=\int_{\Omega}\left[\lambda_{n} u_{n}^{-\gamma}+f\left(z, u_{n}\right)\right] h d z \quad \text { for all } h \in W_{0}^{1, p}(\lambda) \text {, all } n \in \mathbb{N} \text {. }
$$

Suppose that $\left\|u_{n}\right\| \rightarrow \infty$. We set $y_{n}=\frac{u_{n}}{\left\|u_{n}\right\|} n \in \mathbb{N}$. Then $\left\|y_{n}\right\|=1, y_{n} \geq 0$ for all $n \in \mathbb{N}$. So, we may assume that

$$
y_{n} \stackrel{w}{\rightarrow} y \text { in } W_{0}^{1, p}(\Omega) \quad \text { and } \quad y_{n} \rightarrow y \text { in } L^{p}(\Omega) \text { as } n \rightarrow \infty .
$$

From (29) we have

$$
\left\langle A\left(y_{n}\right), h\right\rangle=\int_{\Omega}\left[\frac{\lambda_{n}}{\left\|u_{n}\right\|^{p+\gamma-1}} y_{n}^{-\gamma}+\frac{N_{f}\left(u_{n}\right)}{\left\|u_{n}\right\|^{p-1}}\right] h d z \quad \text { for all } h \in W_{0}^{1, p}(\Omega), \quad n \in \mathbb{N} .
$$

Hypotheses $H(f)(\mathrm{i})$,(ii) imply that

$|f(z, x)| \leq c_{7}\left[1+x^{p-1}\right] \quad$ for almost all $z \in \Omega, \quad$ all $x \geq 0$, and some $c_{7}>0$. 
This growth condition implies that

$$
\left\{\frac{N_{f}\left(u_{n}\right)}{\left\|u_{n}\right\|^{p-1}}\right\}_{n \geq 1} \subseteq L^{p^{\prime}}(\Omega) \text { is bounded. }
$$

Then (32) and hypothesis $H(f)$ (ii) imply that at least for a subsequence, we have

$$
\begin{gathered}
\quad \frac{N_{f}\left(u_{n}\right)}{\left\|u_{n}\right\|^{p-1}} \stackrel{w}{\longrightarrow} \eta_{0}(z) y^{p-1} \quad \text { in } L^{p^{\prime}}(\Omega) \text { as } n \rightarrow \infty, \\
\text { with } \eta \leq \eta_{0}(z) \leq \hat{\eta} \quad \text { for almost all } z \in \Omega \\
\text { (see Aizicovici et al. }[1 \text {, proof of Proposition } 16 \text { ). }
\end{gathered}
$$

In (31) we choose $h=y_{n}-y \in W_{0}^{1, p}(\Omega)$, pass to the limit as $n \rightarrow \infty$, and use (30) and (32). Then

$$
\begin{gathered}
\lim _{n \rightarrow \infty}\left\langle A\left(y_{n}\right), y_{n}-y\right\rangle=0, \\
\Rightarrow y_{n} \rightarrow y \text { in } W_{0}^{1, p}(\Omega) \text { (see Proposition 3), hence }\|y\|=1, \quad y \geq 0 .
\end{gathered}
$$

Therefore, if in (31) we pass to the limit as $n \rightarrow \infty$ and use (34) and (33), then

$$
\begin{gathered}
\langle A(y), h\rangle=\int_{\Omega} \eta_{0}(z) y^{p-1} h d z \quad \text { for all } h \in W_{0}^{1, p}(\Omega), \\
\Rightarrow-\Delta_{p} y(z)=\eta_{0}(z) y(z)^{p-1} \quad \text { for almost all } z \in \Omega,\left.y\right|_{\partial \Omega}=0 .
\end{gathered}
$$

Since $\eta \leq \eta_{0}(z) \leq \hat{\eta}$ for almost all $z \in \Omega$ (see (33) $)$, using Proposition 4 , we have

$$
\tilde{\lambda}_{1}\left(\eta_{0}\right) \leq \tilde{\lambda}_{1}(\eta)<\tilde{\lambda}_{1}\left(\hat{\lambda_{1}}\right)=1 \text {. }
$$

So, from (35) and since $\|y\|=1$ (see (34)), it follows that $y$ must be nodal, a contradiction (see (34)). Therefore,

$$
\left\{u_{n}\right\}_{n \geq 1} \subseteq W_{0}^{1, p}(\Omega) \text { is bounded. }
$$

Hence, we may assume that

$$
u_{n} \stackrel{w}{\longrightarrow} u^{*} \quad \text { in } W_{0}^{1, p}(\Omega) \text { and } \quad u_{n} \rightarrow u^{*} \quad \text { in } L^{p}(\Omega) \text { as } n \rightarrow \infty .
$$

On account of Proposition 5, the sequence $\left\{u_{n}\right\}_{n \geq 1}$ is bounded below. Therefore, $u^{*} \neq 0$. Also, we have

$$
0 \leq\left(u^{*}\right)^{-\gamma} \leq u_{n}^{-\gamma} \leq u_{1}^{-\gamma} \in L^{p^{\prime}}(\Omega) \text { for all } n \in \mathbb{N} .
$$

From (36) and by passing to a subsequence if necessary, we can say that

$$
u_{n}(z)^{-\gamma} \rightarrow u^{*}(z)^{-\gamma} \text { for almost all } z \in \Omega .
$$

From (37), (38) and Problem 1.19 of Gasinski and Papageorgiou [4, we have that

$$
u_{n}^{-\gamma} \stackrel{w}{\longrightarrow}\left(u^{*}\right)^{-\gamma} \quad \text { in } L^{p^{\prime}}(\Omega) \text { as } n \rightarrow \infty \text {. }
$$


If in (29) we choose $h=u_{n}-u^{*} \in W_{0}^{1, p}(\Omega)$, pass to the limit as $n \rightarrow \infty$ and use (39) and the fact that $\left\{N_{f}\left(u_{n}\right)\right\}_{n \geq 1} \subseteq L^{p^{\prime}}(\Omega)$ is bounded, then

$$
\begin{aligned}
& \lim _{n \rightarrow \infty}\left\langle A\left(u_{n}\right), u_{n}-u^{*}\right\rangle=0, \\
\Rightarrow & u_{n} \rightarrow u^{*} \text { in } W_{0}^{1, p}(\Omega) \text { (see Proposition } 3 \text { ). }
\end{aligned}
$$

Finally, in (29) we pass to the limit as $n \rightarrow \infty$ and use (39) and (40). We obtain

$$
\begin{aligned}
& \left\langle A\left(u^{*}\right), h\right\rangle=\int_{\Omega}\left[\lambda^{*}\left(u^{*}\right)^{-\gamma}+f\left(z, u^{*}\right)\right] h d z \quad \text { for all } h \in W_{0}^{1, p}(\Omega), \\
\Rightarrow & u^{*} \in S_{\lambda^{*}} \subseteq \operatorname{int} C_{+} \quad \text { and } \quad \lambda^{*} \in \mathcal{L} .
\end{aligned}
$$

This completes the proof.

We have proved that

$$
\mathcal{L}=\left(0, \lambda^{*}\right]
$$

Proposition 13. If hypotheses $H(f)$ hold and $\lambda \in\left(0, \lambda^{*}\right)$, then problem $\left(P_{\lambda}\right)$ admits at least two positive solutions

$$
u_{\lambda}, \hat{u}_{\lambda} \in \operatorname{int} C_{+}, \quad \hat{u}_{\lambda}-u_{\lambda} \in C_{+} \backslash\{0\} .
$$

Proof. Let $u^{*} \in S_{\lambda^{*}} \subseteq \operatorname{int} C_{+}$(see Proposition 12). Invoking Proposition 10, we can find $u_{\lambda} \in S_{\lambda} \subseteq \operatorname{int} C_{+}$such that

$$
u^{*}-u_{\lambda} \in \operatorname{int} C_{+} .
$$

We consider the Carathéodory function $\tau_{\lambda}(z, x)$ defined by

$$
\tau_{\lambda}(z, x)= \begin{cases}\lambda u_{\lambda}(z)^{-\gamma}+f\left(z, u_{\lambda}(z)\right) & \text { if } x \leq u_{\lambda}(z) \\ \lambda x^{-\gamma}+f(z, x) & \text { if } u_{\lambda}(z)<x .\end{cases}
$$

Recall that $u_{\lambda}^{-\gamma} \in L^{p^{\prime}}(\Omega)$ (see the proof of Proposition [5). We set $T_{\lambda}(z, x)=$ $\int_{0}^{x} \tau_{\lambda}(z, s) d s$ and consider the functional $\tilde{\varphi}_{\lambda}: W_{0}^{1, p}(\Omega) \rightarrow \mathbb{R}$ defined by

$$
\tilde{\varphi}_{\lambda}(u)=\frac{1}{p}\|D u\|_{p}^{p}-\int_{\Omega} T_{\lambda}(z, u) d z \quad \text { for all } u \in W_{0}^{1, p}(\Omega) .
$$

We know that $\tilde{\varphi}_{\lambda} \in C^{1}\left(W_{0}^{1, p}(\Omega)\right)$. Let $K_{\tilde{\varphi}_{\lambda}}=\left\{u \in W_{0}^{1, p}(\Omega): \tilde{\varphi}_{\lambda}^{\prime}(u)=0\right\}$ (the critical set of $\left.\tilde{\varphi}_{\lambda}\right)$. Also, for $u \in W_{0}^{1, p}(\Omega)$, we set

$$
[u)=\left\{v \in W^{1, p}(\Omega): u(z) \leq v(z) \text { for almost all } z \in \Omega\right\} .
$$

Claim 1. $K_{\tilde{\varphi_{\lambda}}} \subseteq\left[u_{\lambda}\right) \cap \operatorname{int} C_{+}$. 
Let $u \in K_{\tilde{\varphi}_{\lambda}}$. We have

$$
\langle A(u), h\rangle=\int_{\Omega} \tau_{\lambda}(z, u) h d z \quad \text { for all } h \in W_{0}^{1, p}(\Omega) .
$$

We choose $h=\left(u_{\lambda}-u\right)^{+} \in W_{0}^{1, p}(\Omega)$. Then

$$
\begin{aligned}
\left\langle A(u),\left(u_{\lambda}-u\right)^{+}\right\rangle & =\int_{\lambda}\left[\lambda u_{\lambda}^{-\gamma}+f\left(z, u_{\lambda}\right)\right]\left(u_{\lambda}-u\right)^{+} d z(\text { see (42) }) \\
& =\left\langle A\left(u_{\lambda}\right),\left(u_{\lambda}-u\right)^{+}\right\rangle\left(\text {since } u_{\lambda} \in S_{\lambda}\right), \\
& \Rightarrow u_{\lambda} \leq u .
\end{aligned}
$$

From (42), (43) and (44), we obtain

$$
\begin{aligned}
& \langle A(u), h\rangle=\int_{\Omega}\left[\lambda u^{-\gamma}+f(z, u)\right] h d z \quad \text { for all } h \in W_{0}^{1, p}(\Omega), \\
\Rightarrow & u \in S_{\lambda} \subseteq \operatorname{int} C_{+} \text {and } u_{\lambda} \leq u, \\
\Rightarrow & u \in\left[u_{\lambda}\right) \cap \operatorname{int} C_{+} .
\end{aligned}
$$

This proves Claim 1 .

Note that $u_{\lambda} \in K_{\tilde{\varphi}_{\lambda}}$. We may assume that

$$
K_{\tilde{\varphi}_{\lambda}} \cap\left[u_{\lambda}, u^{*}\right]=\left\{u_{\lambda}\right\}
$$

or otherwise we already have a second positive smooth solution for problem $P_{\lambda}$ (see (42) ) and so we are done.

We introduce the following Carathéodory function:

$$
\hat{\tau}_{\lambda}(z, x)= \begin{cases}\tau_{\lambda}(z, x) & \text { if } x \leq u^{*}(z), \\ \tau_{\lambda}\left(z, u^{*}(z)\right) & \text { if } u^{*}(z)<x .\end{cases}
$$

We set $\hat{T}_{\lambda}(z, x)=\int_{0}^{x} \hat{\tau}_{\lambda}(z, s) d s$ and consider the $C^{1}$-functional $\hat{\varphi}_{\lambda}: W^{1, p}(\Omega) \rightarrow$ $\mathbb{R}$ defined by

$$
\hat{\varphi}_{\lambda}(u)=\frac{1}{p}\|D u\|_{p}^{p}-\int_{\Omega} \hat{T}_{\lambda}(z, u) d z \quad \text { for all } u \in W_{0}^{1, p}(\Omega) .
$$

This functional is coercive (see (46) ) and sequentially weakly lower semicontinuous. Hence, we can find $\tilde{u}_{\lambda} \in W_{0}^{1, p}(\Omega)$ such that

$$
\begin{aligned}
& \hat{\varphi}_{\lambda}\left(\tilde{u}_{\lambda}\right)=\inf \left\{\hat{\varphi}_{\lambda}(u): u \in W_{0}^{1, p}(\Omega)\right\}, \\
\Rightarrow & \hat{\varphi}_{\lambda}^{\prime}\left(\tilde{u}_{\lambda}\right)=0 \\
\Rightarrow & \left\langle A\left(\tilde{u}_{\lambda}\right), h\right\rangle=\int_{\Omega} \hat{\tau}_{\lambda}\left(z, \tilde{u}_{\lambda}\right) h d z \quad \text { for all } h \in W_{0}^{1, p}(\Omega) .
\end{aligned}
$$

In (477) we choose $h=\left(u_{\lambda}-\tilde{u}_{\lambda}\right)^{+} \in W_{0}^{1, p}(\Omega)$ and $h=\left(\tilde{u}_{\lambda}-u^{*}\right)^{+} \in W_{0}^{1, p}(\Omega)$ and obtain that

$$
\tilde{u}_{\lambda} \in\left[u_{\lambda}, u^{*}\right]
$$


From (46), (47), (48) we infer that

$$
\begin{aligned}
& \tilde{u}_{\lambda} \in K_{\tilde{\varphi}_{\lambda}} \cap\left[u_{\lambda}, u^{*}\right], \\
\Rightarrow & \tilde{u}_{\lambda}=u_{\lambda}(\operatorname{see}(45) .
\end{aligned}
$$

From (42) and (46) it is clear that

$$
\left.\tilde{\varphi}_{\lambda}\right|_{\left[0, u^{*}\right]}=\left.\hat{\varphi}_{\lambda}\right|_{\left[0, u^{*}\right]} .
$$

Also, $u_{\lambda}$ is a minimizer of $\hat{\varphi}_{\lambda}$. Since $u^{*}-u_{\lambda} \in \operatorname{int} C_{+}$(see (41)), it follows that:

$$
\begin{aligned}
& u_{\lambda} \text { is a local } C_{0}^{1}(\bar{\Omega})-\text { minimizer of } \tilde{\varphi}_{\lambda} \\
\Rightarrow & u_{\lambda} \text { is a local } W_{0}^{1, p}(\Omega)-\text { minimizer of } \tilde{\varphi}_{\lambda} . \\
& (\text { see Motreanu et al. }[15, \text { Theorem } 12.18, \text { p. } 409]) .
\end{aligned}
$$

We assume that $K_{\tilde{\varphi}_{\lambda}}$ is finite or otherwise on account of Claim 11, we already have an infinity of positive smooth solutions for problem $P_{\lambda}$ bigger than $u_{\lambda}$ and so we are done. Because of (49), we can find $\rho \in(0,1)$ small such that

$$
\tilde{\varphi}_{\lambda}\left(u_{\lambda}\right)<\inf \left\{\tilde{\varphi}_{\lambda}(u):\left\|u-u_{\lambda}\right\|=\rho\right\}=\tilde{m}_{\lambda}
$$

(see Aizicovici et al. [1, proof of Proposition 29).

Hypothesis $H(f)($ ii) implies that

$$
\tilde{\varphi}_{\lambda}\left(t \hat{u}_{1}\right) \rightarrow-\infty \quad \text { as } t \rightarrow+\infty .
$$

Claim 2. $\tilde{\varphi}_{\lambda}$ satisfies the $C$-condition.

Let $\left\{u_{n}\right\}_{n \geq 1} \subseteq W_{0}^{1, p}(\Omega)$ such that $\left\{\tilde{\varphi}_{\lambda}\left(u_{n}\right)\right\}_{n \geq 1} \subseteq \mathbb{R}$ is bounded and

$$
\left(1+\left\|u_{n}\right\|\right) \tilde{\varphi}_{\lambda}^{\prime}\left(u_{n}\right) \rightarrow 0 \text { in } W^{-1, p^{\prime}}(\Omega)=W_{0}^{1, p}(\Omega)^{*} \text { as } n \rightarrow \infty .
$$

We have

$$
\begin{aligned}
& \left|\left\langle A\left(u_{n}\right), h\right\rangle-\int_{\Omega} \tau_{\lambda}\left(z, u_{n}\right) h d z\right| \\
& \quad \leq \frac{\varepsilon_{n}\|h\|}{1+\left\|u_{n}\right\|} \quad \text { for all } h \in W_{0}^{1, p}(\Omega), \text { with } \varepsilon_{n} \rightarrow 0^{+} .
\end{aligned}
$$

We choose $h=-u_{n}^{-} \in W_{0}^{1, p}(\Omega)$ in (52) and also use (42). Then

$$
\begin{aligned}
& \left\|D u_{n}^{-}\right\|_{p}^{p} \leq c_{8}\left\|u_{n}^{-}\right\| \text {for some } c_{8}>0, \quad \text { and all } n \in \mathbb{N}, \\
\Rightarrow & \left\{u_{n}^{-}\right\}_{n \geq 1} \subseteq W_{0}^{1, p}(\Omega) \text { is bounded. }
\end{aligned}
$$

Suppose that $\left\|u_{n}^{+}\right\| \rightarrow \infty$ and let $y_{n}=\frac{u_{n}^{+}}{\left\|u_{n}^{+}\right\|} n \in \mathbb{N}$. Then $\left\|y_{n}\right\|=1, y_{n} \geq 0$ for all $n \in \mathbb{N}$. So, we may assume that

$$
y_{n} \stackrel{w}{\longrightarrow} y \text { in } W_{0}^{1, p}(\Omega) \quad \text { and } \quad y_{n} \rightarrow y \text { in } L^{p}(\Omega), \quad y \geq 0 .
$$


From (52) and (53), we have

$$
\left|\left\langle A\left(y_{n}\right), h\right\rangle-\int_{\Omega} \frac{N_{\tau_{\lambda}}\left(u_{n}^{+}\right)}{\left\|u_{n}^{+}\right\|^{p-1}} h d z\right| \leq \varepsilon_{n}^{\prime}\|h\| \quad \text { for all } h \in W_{0}^{1, p}(\Omega), \quad \text { with } \varepsilon_{n}^{\prime} \rightarrow 0 .
$$

From (42) and hypothesis $H(f)(\mathrm{ii})$, we have

$$
\begin{aligned}
& \frac{N_{\tau_{\lambda}}\left(u_{n}^{+}\right)}{\left\|u_{n}^{+}\right\|^{p-1}} \stackrel{w}{\longrightarrow} \eta_{0}(z) y^{p-1} \text { in } L^{p^{\prime}}(\Omega) \text { as } n \rightarrow \infty \\
& \text { with } \eta \leq \eta_{0}(z) \leq \hat{\eta} \quad \text { for almost all } z \in \Omega(\text { see (33) }) \text {. }
\end{aligned}
$$

In (55) we choose $h=y_{n}-y \in W_{0}^{1, p}(\Omega)$ and pass to the limit as $n \rightarrow \infty$. Then

$$
\begin{aligned}
& \lim _{n \rightarrow \infty}\left\langle A\left(y_{n}\right), y_{n}-y\right\rangle=0, \\
\Rightarrow & y_{n} \rightarrow y \text { in } W_{0}^{1, p}(\Omega)(\text { see Proposition } 3 \text { ) , hence }\|y\|=1, y \geq 0 .
\end{aligned}
$$

Then passing to the limit as $n \rightarrow \infty$ in (55) and using (56) and (57), we obtain

$$
\begin{aligned}
\langle A(y), h\rangle & =\int_{\Omega} \eta_{0}(z) y^{p-1} h d z \quad \text { for all } h \in W_{0}^{1, p}(\Omega), \\
\Rightarrow-\Delta_{p} y(z) & =\eta_{0}(z) y(z)^{p-1} \quad \text { for almost all } z \in \Omega,\left.y\right|_{\partial \Omega}=0 .
\end{aligned}
$$

As before, using Proposition 4, we have

$$
\begin{aligned}
& \tilde{\lambda}_{1}\left(\eta_{0}\right) \leq \tilde{\lambda}_{1}(\eta)<\tilde{\lambda}_{1}\left(\hat{\lambda}_{1}\right)=1, \\
\Rightarrow & y \text { must be nodal (see (58), (57)), a contradiction (see (57) }) .
\end{aligned}
$$

This proves that $\left\{u_{n}^{+}\right\}_{n \geq 1} \subseteq W_{0}^{1, p}(\Omega)$ is bounded. Hence,

$$
\left.\left\{u_{n}\right\}_{n \geq 1} \subseteq W_{0}^{1, p}(\Omega) \text { is bounded (see (53) }\right) \text {. }
$$

So, we may assume that

$$
u_{n} \stackrel{w}{\longrightarrow} u \text { in } W_{0}^{1, p}(\Omega) \text { and } \quad u_{n} \rightarrow u \text { in } L^{p}(\Omega) \text { as } n \rightarrow \infty .
$$

In (52) we choose $h=u_{n}-u \in W_{0}^{1, p}(\Omega)$, pass to the limit as $n \rightarrow \infty$ and use (59). Then

$$
\begin{aligned}
& \lim _{n \rightarrow \infty}\left\langle A\left(u_{n}\right), u_{n}-u\right\rangle=0, \\
\Rightarrow & u_{n} \rightarrow u \text { in } W_{0}^{1, p}(\Omega) \text { (see Proposition } 3 \text { ). }
\end{aligned}
$$

This proves Claim 2 . 
On account of (50), (51) and Claim 2 we can apply Theorem 1 (the mountain pass theorem) and find $\hat{u}_{\lambda} \in W_{0}^{1, p}(\Omega)$ such that

$$
\begin{aligned}
& \hat{u}_{\lambda} \in K_{\tilde{\varphi}_{\lambda}} \subseteq\left[u_{\lambda}\right) \cap \operatorname{int} C_{+}(\text {see Claim 10), } \\
& \tilde{m}_{\lambda} \leq \tilde{\varphi}_{\lambda}\left(\hat{u}_{\lambda}\right)(\text { see (50) }) \text {, hence } \hat{u}_{\lambda} \neq u_{\lambda} .
\end{aligned}
$$

Therefore $\hat{u}_{\lambda} \in \operatorname{int} C_{+}$is the second positive solution of $P_{\lambda}$ and

$$
\hat{u}_{\lambda}-u_{\lambda} \in C_{+} \backslash\{0\} .
$$

The proof is now complete.

Therefore, we have also proved Theorem A, which is the main result of this paper.

Remark 2. An interesting open problem is whether there is such a bifurcation-type theorem for resonant problems, that is,

$$
\hat{\lambda}_{1} \leq \liminf _{x \rightarrow+\infty} \frac{f(z, x)}{x^{p-1}} \leq \limsup _{x \rightarrow+\infty} \frac{f(z, x)}{x^{p-1}} \leq \hat{\eta} \text { uniformly for almost all } z \in \Omega
$$

or even for the nonuniformly nonresonant problems, that is,

$$
\eta(z) \leq \liminf _{x \rightarrow+\infty} \frac{f(z, x)}{x^{p-1}} \leq \limsup _{x \rightarrow+\infty} \frac{f(z, x)}{x^{p-1}} \leq \hat{\eta} \text { uniformly for almost all } z \in \Omega
$$

with $\eta \in L^{\infty}(\Omega)$ such that

$$
\hat{\lambda}_{1} \leq \eta(z) \quad \text { for almost all } z \in \Omega, \quad \eta \not \equiv \hat{\lambda}_{1} .
$$

In both cases it seems to be difficult to show that $\lambda^{*}<\infty$. Additional conditions on $f(z, \cdot)$ might be needed.

\section{Acknowledgments}

The authors wish to thank the referee for his/her remarks and suggestions. This research was supported by the Slovenian Research Agency grants P1-0292, J1-8131, J1-7025, N1-0064, and N1-0083. V. D. Rădulescu acknowledges the support through a grant of the Romanian Ministry of Research and Innovation, CNCS-UEFISCDI, project number PN-III-P4-ID-PCE-2016-0130, within PNCDI III.

\section{References}

[1] S. Aizicovici, N. S. Papageorgiou and V. Staicu, Degree Theory for Operators of Monotone Type and Nonlinear Elliptic Equations with Inequality Constraints Vol. 196 (Memoirs of the American Mathematical Society, 2008), vi+70 pp.

[2] M. Coclite and G. Palmieri, On a singular nonlinear Dirichlet problem, Comm. Partial Diff. Equ. 14 (1989) 1315-1327.

[3] L. Gasinski and N. S. Papageorgiou, Nonlinear Analysis (Chapman \& Hall/CRC, Boca Raton, FL, 2006). 
[4] L. Gasinski and N. S. Papageorgiou, Exercises in Analysis, Part 2: Nonlinear Analysis (Springer, Cham, 2016).

[5] M. Ghergu and V. D. Rădulescu, Singular elliptic problems with two parameters, J. Diff. Equ. 195 (2003) 520-536.

[6] M. Ghergu and V. D. Rădulescu, Singular Elliptic Problems: Bifurcation and Asymptotic Analysis (Clarendon Press, Oxford, UK, 2008).

[7] J. Giacomoni, I. Schindler and P. Takač, Sobolev versus Hölder local minimizers and existence of multiple solutions for a singular quasilinear equation, Ann. Sci. Norm. Super. Pisa, Cl. Sci. 5(6) (2007) 117-158.

[8] M. Guedda and L. Véron, Quasilinear elliptic equations involving critical Sobolev exponents, Nonlinear Anal. 13 (1989) 879-902.

[9] E. Hewitt and K. Stromberg, Real and Abstract Analysis (Springer-Verlag, New York, 1975).

[10] N. Hirano, C. Saccon and N. Shioji, Brezis-Nirenberg type theorems and multiplicity of positive solutions for a singular elliptic problem, J. Diff. Equ. 245 (2008) 19972037.

[11] A. V. Lair and A. W. Shaker, Entire solution of a singular semilinear elliptic problem, J. Math. Anal. Appl. 200 (1996) 498-505.

[12] A. C. Lazer and P. J. McKenna, On a singular nonlinear elliptic boundary value problem, Proc. Amer. Math. Soc. 11 (1001) 721-730.

[13] G. Lieberman, Boundary regularity for solutions of degenerate elliptic equations, Nonlinear Anal. 12 (1988) 1203-1219.

[14] S. A. Marano and N. S. Papageorgiou, Positive solutions to a Dirichlet problem with $p$-Laplacian and concave-convex nonlinearity depending on a parameter, Comm. Pure Appl. Anal. 12 (2013) 815-829.

[15] D. Motreanu, V. Motreanu and N. S. Papageorgiou, Topological and Variational Methods with Applications to Nonlinear Boundary Value Problems (Springer, New York, 2014).

[16] N. S. Papageorgiou, V. D. Rădulescu and D. D. Repovš, Pairs of positive solutions for resonant singular equations with the p-Laplacian, Electr. J. Diff. Equ. 249 (2017) 22 .

[17] N. S. Papageorgiou, V. D. Rădulescu and D. D. Repovš, $(p, 2)$-equations asymmetric at both zero and infinity, Adv. Nonlinear Anal. 7(3) (2018) 327-351.

[18] N. S. Papageorgiou and G. Smyrlis, A bifurcation-type theorem for singular nonlinear elliptic equations, Methods Appl. Anal. 22 (2015) 147-170.

[19] K. Perera and Z. Zhang, Multiple positive solutions of singular $p$-Laplacian problems by variational methods, Bound. Value Problems 2009 (2005) 377-382.

[20] M. Struwe, Variational Methods (Springer-Verlag, Berlin, 1990).

[21] Y. Sun, S. Wu and Y. Long, Combined effects of singular and superlinear nonlinearities in some singular boundary value problems, J. Diff. Equ. 176 (2001) 511-531. 